\title{
artigo
}

Paugartner, L.M.; Dellanhese, A.P.F.; Amorin, B.; Fernandes, M.T.C.

Reações adversas à doação de sangue: uma revisão integrativas

\section{Reações adversas à doação de sangue: uma revisão integrativa}

\author{
Adverse reactions to blood donation: an integrative review \\ Reacciones adversas a la donación de sangre: una revisión integrativa
}

\begin{abstract}
RESUMO
Objetivo: Identificar as reações adversas mais prevalentes à doação de sangue. Métodos: Revisão bibliográfica integrativa, com artigos científicos indexados nas bases de dados PUBMED e LILACS através dos descritores: "adverse effects" e "blood donors". Utilizou-se os operadores boleanos "AND" e "OR". Os artigos resgatados foram aqueles publicados entre 2013 e 2018.0 estudo foi realizado de março a maio de 2018. Resultados: Foram encontrados 119 artigos através das buscas nas bases de dados, restando 13 artigos para leitura na íntegra, sendo incluídos somente 04 a partir da literatura cinza por relação com o tema. Constatou-se uma baixa ocorrência de reações adversas em relação à proporção de doadores. Observa-se predomínio das reações adversas de grau leve, seguida pelas reações de grau moderado e grave. Conclusões: Conhecer os sinais e sintomas dos eventos adversos proporcionará o desenvolvimento de estratégias de gerenciamento para controle da reação adversa à doação de sangue.
\end{abstract}

DESCRITORES: Efeitos Adversos; Doadores de Sangue.

\section{ABSTRACT}

Objective: Identify the most predominant adverse reactions to blood donation. Method: Integrative bibliographic review, with scientific articles indexed in the PUBMED and LILACS databases through the descriptors: "adverse effects" and "blood donors". Use the Boolean operators "and" and "or". The studies rescued were those published between 2013 and 2018. The study was carried out from March to May 2018. Results: 119 articles were found through the searches in the databases, leaving 13 articles for reading in full, being included only 4 from the gray literature by relation to the topic. There was a low occurrence of adverse reactions concerning the proportion of donors. Mild adverse reactions are predominant, followed by moderate and severe reactions. Conclusions: Knowing the signs and symptoms of adverse events will provide the development of a management strategy to control an adverse reaction to blood donation.

DESCRIPTORS: Adverse Effects; Blood Donors.

\section{RESUMEN}

Objetivo: Identificar las reacciones adversas más prevalentes a la donación de sangre. Métodos: Revisión bibliográfica integrativa, con artículos científicos indexados en las bases de datos PUBMED y LILACS a través de los descriptores: "adverse effects" y "blood donors". Se utilizaron los operadores booleanos "and" y "or". Los estudios rescatados fueron los publicados entre 2013 y 2018. El estudio se llevó a cabo de marzo a mayo de 2018. Resultados: Se encontraron 119 artículos mediante búsquedas en las bases de datos, quedando 13 artículos para leer en su totalidad, con solo 4 a partir de literatura gris relacionada con el tema. Hubo una baja incidencia de reacciones adversas en relación con la proporción de donantes. Hay un predominio de reacciones adversas leves, seguidas de reacciones moderadas y graves. Conclusiones: Conocer los signos y síntomas de los eventos adversos permitirá desarrollar estrategias de manejo para controlar las reacciones adversas a la donación de sangre.

DESCRIPTORES: Efectos Adversos; Donantes de Sangre.

RECEBIDO EM: 15/08/2020 APROVADO EM: 25/09/2020

\section{Luciana Medeiros Paungartner}

Enfermeira - Pós Graduação em UTI Geral e Gestão da Assistência Intensiva ao Paciente Crítico - Instituto Faveni - em andamento.

ORCID: 0000-0003-1983-1114 


\section{Amanda Pereira Ferreira Dellanhese}

Enfermeira. Doutora em Saúde da Criança e do Adolescente pela Universidade Federal do Rio Grande do Sul. Docente Colaboradora da Residência Integrada Multiprofissional em Saúde Coletiva da Universidade Federal do Rio Grande do Sul.

ORCID: 0000-0002-1515-9693

\section{Bruna Amorin}

Pós-Doutora em Terapia Celular pela Universidade Federal do Rio Grande do Sul. Pesquisadora do Centro de Processamento Celular Avançado do Hospital de Clínicas de Porto Alegre e Professora da Cesuca Faculdade Inedi.

ORCID: 0000-0002-8912-9171

\section{Morgana Thaís Carollo Fernandes}

Enfermeira. Pós-Doutoranda em Saúde da Criança pela Pontifícia Universidade Católica do Rio Grande do Sul (PUCRS), bolsista pela University of Toronto (UofT). Professora Colaboradora da Residência Multiprofissional em Saúde da Criança e Pesquisadora Associada do Programa de Extensão e Pesquisa em Saúde Urbana, Ambiente e Desigualdades da Universidade Federal do Rio Grande do Sul (UFRGS).

ORCID: 0000-0002-7989-294X

\section{INTRODUÇÃO}

A doação de sangue tem um papel importante nas práticas assistenciais. Acredita-se que em média 250 milhões de pessoas são expostas a pelo menos uma emergência todos os anos, sendo em algumas, a transfusão de hemocomponentes fundamental para a manutenção a vida ${ }^{1}$. No Brasil de 2010 a 2016 aproximadamente 25 milhões de pessoas foram candidatas à doação, com a média anual de cerca de quatro milhões. Destes, 19,2 doadores de 1.000 habitantes da população brasileira foram considerados aptos à doação de sangue 2 . A taxa de doadores de sangue voluntários do Brasil, o coloca acima dos países de média renda que possuem uma taxa de 11,7 doadores de 1.000 habitantes, porém muito abaixo da metade dos países de alta renda com 36,8 doadores de 1.000 habitantes. Sendo assim, sugere-se adotar estratégias para a captação de doadores ${ }^{3}$.

A doação de sangue na maioria dos casos transcorre sem qualquer intercorrência, entretanto, ocasionalmente, alguns doadores podem apresentar reações adversas durante ou após o término da coleta ${ }^{4}$. Caracteriza-se como reação adversa à doação de sangue a resposta indesejada durante ou após a doaçãó . Sendo estas classificadas em intercorrências clínicas leves como mal-estar geral, ansiedade, sudorese, palidez e tontura, em moderadas quando acrescidas de náusea seguida de vômito, períodos rápidos de inconsciências e diminuição constante da pressão arterial (PA sistólica $60 \mathrm{mmHg}$ ou menor) e intercorrências clínicas graves quando somam-se as con- vulsões ou perda súbita da consciência 5 .

Essas reações devem ser investigadas e registradas, visando ações corretivas e preventivas pela equipe técnica onde são realizadas as coletas ${ }^{3}$. Além disso, se faz necessária a notificação das reações adversas pelo serviço onde ocorreu, para o sistema nacional de vigilância sanitária, de acordo com as legislações vigentes ou diretrizes específicas do Sistema Nacional de Hemovigilância ${ }^{6}$.

Com o objetivo de minimizar as reações adversas à doação de sangue, os doadores são avaliados por meio de uma entrevista individual denominada triagem clínica ${ }^{5}$ A triagem clínica deve ser realizada de forma rigorosa classificando em inapto o candidato que possivelmente poderá apresentar uma resposta indesejada a doação de sangue ${ }^{7}$.

Essa triagem consiste na avaliação clínica e epidemiológica, do estado atual de saúde, dos hábitos e comportamentos do doador, assim determinando se o mesmo está apto a doar sangue sem que haja prejuízo à sua saúde e deve ser feita sempre quando o doador irá realizar o ato de doar o sangue8. Percebe-se que o preparo dos profissionais da saúde nos serviços de hemoterapia para lidarem com essas situações é de extrema importância, visto a necessidade de saber manejar a situação, garantindo segurança e bem-estar dos doadores e notificar a reação após o acontecimento ${ }^{4,5,9}$.

Dentre estes profissionais, destaca-se a equipe de enfermagem, uma vez que, está em contato direto com os doadores durante o processo de doação de sangue, sendo assim necessitam estarem preparados para o primeiro atendimento objetivando a estabilização do quadro clínico ${ }^{5,10}$. Sendo assim, objetivou-se identificar as reações adversas mais prevalentes à doação de sangue.

\section{MÉTOdO}

Foi realizada uma revisão integrativa, que consiste em um método de pesquisa, que utiliza a sistematização e resultados de uma pesquisa em base de dados, relevante para a assistência da saúde, permitindo a síntese de múltiplos estudos publicados e possibilidade de conclusões gerais a respeito de uma particular área de estudo ${ }^{11,12}$.

Em relação à relevância das escolhas dos estudos estabeleceu-se como critério de seleção, 1) identificação do tema e seleção da hipótese ou questão de pesquisa para a elaboração da revisão integrativa; 2) estabelecimento de critérios para inclusão e exclusão de estudos/amostragem ou busca na literatura; 3) definição das informações a serem extraídas dos estudos selecionados/ categorização dos estudos; 4) avaliação dos estudos incluídos na revisão integrativa; 5) interpretação dos resultados e 6) apresentação da revisão/síntese do conhecimento ${ }^{11}$.

Para análise dos dados foi utilizado o Prisma ${ }^{\circ}$ que consiste em um check list de avaliação da qualidade dos estudos13. A pergunta norteadora da pesquisa foi: "quais as reações adversas mais prevalentes à doação de sangue?”. As bases de dados eletrônicas utilizadas foram: LILACS e PUBMED. Utilizou-se os seguintes descritores 


\section{artigo}

estratégia de busca: "adverse effects" AND "blooddonors". A busca ocorreu no período de março a maio de 2018. Foram resgatados os estudos publicados na íntegra no período de 2013 a 2018, com texto completo disponível em português e inglês. Incluiu-se estudos, de delineamento transversal e coorte. Para fins de inclusão excessiva de artigos, todos unitermos foram selecionados e identificados nos três principais campos de pesquisa (title, abstract e keywords). Desta forma, os unitermos deveriam, obrigatoriamente, constar em pelo menos um dos três campos de pesquisa. Todo processo de seleção foi realizado na presença e concordância de dois pesquisadores.

\section{RESULTADOS}

Foram localizados 119 artigos a partir das buscas nas bases de dados. Após leitura dos títulos e resumos restaram 13 artigos. Posteriormente após leitura na íntegra nenhum estudo com relação específica com o tema foi localizado, entretanto, a partir da análise da literatura cinza quatro artigos foram incluídos na presente revisão.

Dos estudos selecionados, dois eram brasileiros, um indiano e um italiano. A Tabela 1 apresenta o tipo de estudo realizado, correspondendo a metade coorte e a outra transversal, ou seja, todos os estudos apresentaram nível de evidência IV. Constatou-se uma baixa ocorrência de reações adversas em relação a proporção de doadores, sendo 1.369 (3\%) de 45.584 mil; 113 (0,6\%) de 19.045 mil; 16.129 (2,2\%) de 724.861 mil; e 63 (1,2\%)de 4.906 mil, respectivamente $5,9,13,14$.

Observa-se predomínio das reações adversas de grau leve, 1269 (92,6\%), 79 (0,4\%), $15.239(94,4 \%)$ e $59(1 \%)^{5,9,14,15}$, sendo as mais frequentes mal-estar geral, tontura, palidez, agitação e sudorese. Em seguida às reações de grau moderado, 79 (5,8\%), 9 $(0,05 \%), 745$ (4,6\%), com registro de perda transitória do nível de consciência e náuseas seguidas de vômitos ${ }^{5,9,15}$, tendência a lipotimia, escurecimento facial e hipotensão ${ }^{5}$. Por fim, às reações graves, 21 (1,6\%),1 (0,005\%), $145(0,9 \%)$ e $4(0,2 \%)^{5,9,14,15}$.

\section{DISCUSSÃO}

Evidencia-se uma baixa prevalência de reaçôes adversos relacionados a doação de sangue. Apesar de poucos riscos para a saúde dos doadores, podem impactar aos que apresentaram reações, ressaltando a importância do registro das informações, como condutas prévias a doação e tipo da reação adversa de forma preventiva ${ }^{4}$.

Para fins de rastreamento, todos os serviços de hemoterapia devem fornecer informaçōes sobre a doação de sangue e possíveis intercorrências durante ou após o processo. Orienta-se um método de fácil comunicação do doador e serviço para eventos tardios, com o propósito de serem notificados mesmo horas ou dias após a doação, realizando também o registro da conduta prestada ${ }^{15}$. Percebe-se que é de suma importância o conhecimento dos profissionais que realizam o atendimento tanto na fase de rastreamento durante a aplicação do questionário clínico como na doação propriamente dita.

Com o livre acesso aos registros anteriores, é possível agregar informações em futuros documentos para realizar uma triagem clínica

Tabela 1: Caracterização de estudos elegíveis da base de dados Prisma, incluídos na revisão integrativa, São Paulo-SP, 2020.

Estudo

Amostra

Nível de Evidência e Delineamento

Silva et al., Texto Contexto Enferm, 2014.5 Nursing care procedures in response to adverse events to blood donation.

Goncalez et al., Author manuscript, 2013.15 Vasovagal reactions in whole blood donors at 3 REDS-II blood centers in Brazil.

Pathak et al., Blood Transfus, 2011.9 Adverse reactions in whole blood donors: an Indian scenario.

Crocco; D'Elia, Blood Transfus, 2007.14 Adverse reactions during voluntary donation of blood and/or blood components.
$45.584 \quad$ (IV) Coorte

19.045 (IV) Coorte

4.906 (IV) Transversal
Identificar os tipos de eventos adversos e suas manifestações clínicas, analisando as condutas de enfermagem que foram adotadas frente a essas reações.

Avaliar a frequência e os fatores associados às reações vaso-vagais em doadores de sangue.

Estimar a frequência e o tipo de evento adverso ocorrido com doadores de sangue e avaliar as práticas que ajudariam a minimizá-los.

Estimar a frequência e o tipo de evento adverso a doação de sangue quanto a sua gravidade.

\section{Principais Resultados}

Ocorreram 1369 (3\%) eventos adversos, sendo $1269(92,6 \%), 79$ $(5,8 \%)$ e $21(1,6 \%)$ leves, moderados e graves respectivamente. As manifestações clínicas graves foram convulsão, contratura muscular e lipotimia.

Foram registradas 16.129 (2,2\%) reações vaso-vagais, sendo $15.239(94,4 \%)$ leves, $745(4,6 \%)$ moderadas e $145(0,9 \%)$ graves.

Constatou-se $113(0,6 \%)$ eventos adversos. Sendo $79(0,4 \%)$ leves, $9(0,05 \%)$ moderados e $1(0,005$ $\%$ ) graves.

Da amostra analisada, 63 (1,2\%), sofreram algum tipo de reação adversa sendo $59(1 \%)$ reações leves e $4(0,2 \%)$ reações graves sendo estas, vômitos, perda de consciência e síncope convulsiva. 
eficaz diminuindo a ocorrência desses eventos $^{15}$. Como limitações, destaca-se a escassez de estudos, visto que, não há muitas publicaçôes científicas acerca das reações adversas à doação de sangue, principalmente no Brasil. Se faz necessário, novos estudos a fim de se conhecer os principais motivos em que os doadores apresentam essas respostas indesejadas e condutas de manejo adotadas para que, dessa forma, se possa intervir de maneira mais precisa. Ressalta-se quanto a qualidade dos estudos incluídos como as inconsistências amostrais destacadas nas variáveis analisadas.
Para prevenção de novas reações adversas sugere-se diminuir o tempo de espera para a doação, evitar jejum superior a quatro horas e fornecer água e lanche antes da doação. Além disso, deve-se identificar doadores ansiosos e assegurar-lhes o maior conforto e atentar a equipe para uma atenção assídua. Evitar a posição sentada ou semi-sentada durante a doação e atentar aos primeiros sinais e sintomas $^{9}$ 16, 17. A importância da notificação deve ser sempre reforçada com os profissionais que realizam o atendimento, visto que, essa constitui-se como uma ferramenta reativa para a ges- tão de risco indispensável para a hemovigilância e monitoramento dos eventos adversos ${ }^{6}$.

\section{CONCLUSÃO}

Conclui-sequea prevalência de reações adversasé baixa e as que mais prevalentes são as reações leves, mostrando-se assim o baixo risco envolvido no processo de doação de sangue. Espera-se que o estudo possa subsidiar o desenvolvimento de produções literárias futuras, objetivando minimizar esses eventos, com o foco na segurança dos doadores envolvidos nesse processo.

\section{REFERÊNCIAS}

1. OPAS. Organización Panamericana de la Salud e Organización Mundial de la Salud. Suministro de sangre para transfusionesenlos países delatinoaméricay del caribe 2014 y 2015. Disponível em: http://iris.paho.org/xmlui/bitstream/handle/123456789/34082/97 89275319581-spa.pdf?sequence=1\&isAllowed=y.

2. BRASIL. Agência Nacional de Vigilância Sanitária. $5^{\circ}$ Boletim de Produção Hemoterápica. Ministério da Saúde: Brasilia, 2018. Disponivel em:http://portal.anvisa.gov.br/ documents $/ 33840 / 2817173 / 5 \%$ C $2 \% B A+B o l e t i m+d e+P r o-$ du\%C3\%A7\%C3\%A30+Hemoter\%C3\%A1pica/d3f3788d-a907-4180a642-4e2e22ed53ce >.

3. BRASIL. Agência Nacional de Vigilância Sanitária.Portaria No 158, de 4 de fevereiro de 2016.Ministério da Saúde: Brasilia, 2018. Disponivel em:http://bvsms.saude.gov.br/bvs/saudelegis/gm/2016/ prt0158_04_02_2016.html.

4. BRASIL. Agência Nacional de Vigilância Sanitária. Marco Conceitual e Operacional de Hemovigilância: Guia para a Hemovigilância no Brasil, Ministério da Saúde: Brasilia, 2015. Disponível em:http://portal. anvisa.gov.br/documents/33868/404938/guia_hemovigilancia15. pdf/495fd617-5156-447d-ad22-7211cdbab8a7.

5. Silva KFN, Barichello E, Mattia AL, Barbosa MH. Condutas de enfermagem adotadas diante dos eventos adversos à doação de sangue. Texto contexto - enferm. Florianópolis, 2014; 23(3):688-95, Set. . Disponivel em: http://www.scielo.br/pdf/tce/v23n3/pt_0104-0707tce-23-03-00688.

6. BRASIL. Ministério da Saúde. Documento de referência para o Programa Nacional de Segurança do Paciente: Ministério da Saúde Brasilia (DF) 2014. Disponivel em: http://bvsms.saude.gov.br/bvs/ publicacoes/documento_referencia_programa_nacional_seguranca.pdf

7. De Azevedo AS, Nogueira CSC, Artiles CB, Domigues CF, Alves CN, Malheiros GN, et al. Fatores da triagem clínica que impedem a doação de sangue. Revista Científica da Faculdade de Medicina de Campos.2015; 10(2):7-11. ISSN 1980-7813. Disponível em: http://www.fmc.br/wp-content/uploads/2016/04/Rev-CientFMC-2-2015-7-11-1.pdf

8. Pinho AMD, Lopes MID, Lima MJR, Castro V. Triagem clínica de do- adores de sangue. In: (Ed.). Triagem clínica de doadores de sangue, 2001. Disponivel em: http://bvsms.saude.gov.br/bvs/publicacoes/ cd07_20.pdf

9. Pathak C, Pujani M, Pahuja S, Jain M. Adverse reactions in whole blood donors: an Indian scenario. Blood Transfusion.2011; 9(1):46. Disponível em: https:/www.ncbi.nlm.nih.gov/pmc/articles/ PMC3021397/

10. Schöninger N, Duro CLM. Atuação do enfermeiro em serviço de hemoterapia. Ciência, cuidado e saúde 2010; 9(2):317-24. ISSN 1984-7513. Disponivel em: http://periodicos.uem.br/ojs/index.php/ CiencCuidSaude/article/viewFile/11239/6082

11. Mendes KDS, Silveira RCCP, Galvão CM. Revisão integrativa: método de pesquisa para a incorporação de evidências na saúde e na enfermagem. Texto contexto - enferm. 2008; 17(4):758-64, Dec. . Disponivel em: http://www.scielo.br/pdf/tce/v17n4/18.

12. De Souza MT,Da Silva MD,De Carvalho R. Revisão integrativa: 0 que é e como fazer. Einstein.2010; 8(1):102-6. Disponivel em: http:// www.scielo.br/pdf/eins/v8n1/pt_1679-4508-eins-8-1-0102

13. Prisma, 2015. Disponível em: http://www.prisma-statement.org/ 14. Gonçalez TT, Sabino EC, Schlumpf KS, Wright DJ, Leao S, Sampaio $D$, et al. Vasovagal reactions in whole blood donors at three REDS II blood centers in Brazil. Transfusion.2012; 52(5):1070-8, ISSN 15372995. Disponivel em: https://www.ncbi.nlm.nih.gov/pmc/articles/ PMC3687805/

15. Crocco A, D'elia D. Adverse reactions during voluntary donation of blood and/or blood components. A statistical-epidemiological study. Blood transfusion.2007; 5(3):143. Disponivel em: https:/www.ncbi. nlm.nih.gov/pmc/articles/PMC2535889/

16. BRASIL. Ministério da Saúde. Portaria $n^{\circ} 158$, de 4 de fevereiro de 2016. Redefine o regulamento técnico de procedimentos hemoterápicos. Disponivel em:http://www.hemocentro.unicamp.br/ dbarquivos/portaria_ms_n_158_de_04_de_fevereiro_2016.pdf.

17. WHO. World Health Organization. Diretrizes da OMS para a tiragem de sangue: boas práticas em flebotomia, 2010. Disponível em: http://www.who.int/infection-prevention/publications/Phlebotomy-portuges_web.pdf 